\title{
SR-BI associates with ABCG1 and inhibits ABCG1-mediated cholesterol efflux from cells to high-density lipoprotein 3
}

\author{
Guohua Song $^{1 \dagger}$, Chuanlong Zong ${ }^{1 \dagger}$, Qian Liu', Yanhong Si ${ }^{1}$, Jie Liư ${ }^{2}$, Wei Li ${ }^{1}$, Ping Zhu ${ }^{2,4^{*}}$ and Shucun Qin ${ }^{1,3^{*}}$
}

\begin{abstract}
Background: The single and combined effects of scavenger receptor-BI (SR-BI), ATP-binding cassette transporter (ABC) A1 and G1 on cholesterol efflux from Chinese Hamster Ovary (CHO) cells were investigated.

Results: When apolipoproteinA-I (apoA-I) was used as an acceptor, ABCA1 overexpression led to an increase in total cholesterol (TC) in medium which is attributable to a 2-fold increase in free cholesterol (FC) content. When high-density lipoprotein 3 (HDL3) was used as an acceptor, SR-BI overexpression not only promoted FC efflux, but also promoted the uptake of cholesteryl ester (CE) into cells, resulting in no TC varieties in medium. Overexpression of ABCG1 increased both the FC and CE levels in medium. However, when apoA-I and HDL3 were both used as acceptors, coexpression of SR-BI has no effect on ABCA1-mediated increased FC and TC accumulation in medium. Interestingly, coexpression of SR-BI with ABCG1 blocked the ABCG1-mediated cholesterol efflux to HDL3, mostly by promoting the reuptake of $C E$ from the medium. Furthermore, co-immunoprecipitation experiments revealed that SR-Bl interacted with ABCG1 in BHK cells overexpressing ABCG1 and SR-BI.
\end{abstract}

Conclusions: We found SR-BI associates with ABCG1 and inhibits ABCG1-mediated cholesterol efflux from cells to HDL3.

\section{Background}

Reverse cholesterol transport (RCT) is assumed to play a critical role in the pathogenesis of atherosclerosis [1]. Cellular cholesterol efflux, by which cholesterol is transported from peripheral cells to high-density lipoprotein (HDL) acceptor molecules, is the first step of RCT [2]. ATPbinding cassette transporter $(\mathrm{ABC}) \mathrm{A} 1, \mathrm{G} 1$ and scavenger receptor-BI (SR-BI) are three important mediators of cholesterol efflux. ABCA1 exports cholesterol and phospholipid to lipid-poor apolipoproteins and initiates HDL formation by lipidating apolipoproteinA-I (apoA-I) [3]. However, ABCA1 interacts poorly with HDL2 and HDL3 particles, which constitute the bulk of the plasma HDL. Another two cell surface transporters, ABCG1 and SR-BI, have been described to export cholesterol to phospholipid-containing acceptors, including HDL. SR-BI

\footnotetext{
*Correspondence: shucunqin@hotmail.com; zhuping301@yahoo.com.cn ${ }^{\dagger}$ Equal contributors

'Key Laboratory of Atherosclerosis in Universities of Shandong; Institute of Atherosclerosis, Taishan Medical University, Taian, China

${ }^{2}$ PLA General Hospital, Beijing, China

Full list of author information is available at the end of the article
}

stimulates the bidirectional flux of cholesterol between cells and lipoproteins [4], while ABCG1 has been shown to promote free cholesterol (FC) efflux from macrophage to HDL and increase the accumulation of cholesteryl ester (CE) in medium as a result of lecithin-cholesterol acyltransferase (LCAT)-mediated cholesterol esterification [5].

It has been reported that mice lacking ABCA1 expression in macrophages developed accelerated atherosclerosis [6]. Conversely, mice with macrophage overexpression of ABCA1 have decreased atherosclerosis [7]. Mice lacking ABCG1 have evidence of lipid accumulation in certain tissue macrophages [8,9]. Furthermore, transplantation of bone marrow from SR-BI deficient (SR-BI $-/-$ ) mice into LDL receptor $-/-$ or apoE $-/-$ mice have shown an increase in atherosclerosis [10-12]. Taken together, these findings suggest that all three transporters involving macrophage ABCA1, ABCG1 and SR-BI may exert protective functions in atherosclerosis. In addition, many reports have indicated a synergistic relationship between $A B C A 1$ and $A B C G 1$ in peripheral tissues, where ABCA1 lipidates any lipid-poor/free apoA-I to generate nascent or

\section{Ciomed Central}


pre- $\beta$-HDL. These particles in turn may serve as substrates for ABCG1-mediated cholesterol export [13-15]. However, the relationships between SR-BI and the two $\mathrm{ABC}$ transporters are not well understood. The current study was undertaken to compare the roles of SR-BI and the two $\mathrm{ABC}$ transporters in promoting mass cholesterol efflux and determine a possible interaction between the SR-BI- and ABC transporter-mediated cholesterol efflux pathways in vitro. We found coexpression of SR-BI with ABCG1 blocked the ABCG1-mediated cholesterol efflux to HDL3, mostly by promoting the reuptake of $\mathrm{CE}$ from the medium. Furthermore, co-immunoprecipitation experiments revealed that SR-BI interacted with ABCG1 in BHK cells overexpressing ABCG1 and SR-BI, which might play roles in maintaining cellular cholesterol homeostasis.

\section{Results}

The single effects of SR-BI, ABCA1 and ABCG1 on cellular cholesterol efflux

It has been reported that lipid-free apoA-I is the lipid acceptor of ABCA1-mediated cholesterol efflux [16-18], while SR-BI [19] and ABCG1 $[8,20]$ were identified as being mediators of cholesterol efflux to mature HDL, but not to lipid-free apoA-I in vitro. Therefore, we used apoA-I as lipid acceptors in ABCA1 transfected cells and HDL3 as lipid acceptors in SR-BI or ABCG1 transfected cells. As shown in Figure 1, SR-BI overexpression not only increased the accumulation of FC (Figure 1A), but also decreased the accumulation of $\mathrm{CE}$ in medium (Figure 1B), resulting in no total cholesterol (TC) varieties in medium (Figure 1C). These data gave us a clue that SR-BI overexpression might not only promote FC efflux, but also promote the uptake of CE into cells. In addition, $\mathrm{ABCA} 1$ overexpression led to an increase in $\mathrm{TC}$ in medium (Figure $1 \mathrm{C}$ ), in large part attributable to a 2-fold increase in FC content (Figure 1A), while overexpression of ABCG1 increased both the FC (Figure 1A) and $\mathrm{CE}$ (Figure 1B) levels in medium.

\section{The effects of SR-BI on ABCA1-mediated cellular cholesterol efflux}

Because ABCA1 and ABCG1 act in a cooperative fashion to promote cellular cholesterol efflux [13], we next determined whether SR-BI might facilitate ABCA1mediated cellular cholesterol efflux. Thus, we coexpressed SR-BI and ABCA1 in CHO cells and determined the cholesterol efflux to apoAI and HDL3 (apoA-I and HDL3 were both used as acceptors). As shown in Figure 2, coexpression of SR-BI with ABCA1 has no effect on $\mathrm{ABCA} 1$-mediated increased $\mathrm{FC}$ (Figure 2A) and TC (Figure 2B) accumulation in medium. Also, the $\mathrm{CE}$ levels in medium were not changed in SR-BI and ABCA1 cotransfected cells compared with cells overexpressing
ABCA1 alone (Figure 2C). These data indicated that SR-BI overexpression has no effect on ABCA1-mediated cellular cholesterol efflux to apoAI and HDL3 in vitro. Moreover, the expression levels of ABCA1 were similar in cells transfected with $A B C A 1$ alone or $A B C A 1$ plus SR-BI (Figure 2D), which indicates that our findings were not attributable to a difference in ABCA1 expression levels.

\section{The effects of SR-BI on ABCG1-mediated cellular cholesterol efflux}

To evaluate the role of SR-BI on ABCG1-mediated cholesterol efflux, we coexpressed SR-BI and ABCG1 in $\mathrm{CHO}$ cells and determined the cholesterol efflux to HDL3. As shown in Figure 3, coexpression of SR-BI with ABCG1 blocked the ABCG1-mediated cholesterol efflux to HDL3, and it seems that this effect of SR-BI was mostly achieved by promoting the reuptake of CE from the medium. This was similar to previous findings, in which it was indicated SR-BI inhibits ABCG1-stimulated $\mathrm{CE}$ accumulation in medium in an LCAT-dependent manner by using HEK293 cell model [21].

\section{SR-BI co-immunoprecipitated with ABCG1 in BHK cells overexpressing SR-BI and ABCG1}

Co-immunoprecipitation experiments with cell lysates from SR-BI and ABCG1 co-transfected BHK cells were performed to confirm whether SR-BI and ABCG1 were present in the same complex. The immunoprecipitates were then analyzed by western blot with antibodies against SR-BI. As shown in Figure 4, SR-BI were detected in the anti-ABCG1 immunoprecipitates from the cell lysates of transfected BHK cells. These results suggested that SR-BI associates with ABCG1 in BHK cells overexpressing SR-BI and ABCG1.

\section{Discussion}

The purpose of the present study was to compare the roles of SR-BI, ABCA1 and ABCG1 singly or together in mediating cholesterol efflux from cells in vitro. We found that SR-BI associates with ABCG1 and inhibits ABCG1-mediated cholesterol efflux from cells to HDL3, and that overexpression of SR-BI has no effect on ABCA1-mediated cellular cholesterol efflux to apoAI and HDL3 in vitro.

It has been reported that $\mathrm{ABCA} 1$ exports cholesterol and phospholipid to lipid-poor apolipoproteins, and interacts poorly with HDL2 and HDL3 particles [3]. ABCG1 and SR-BI have been described to export cholesterol to phospholipid-containing acceptors, including HDL [4]. In our study, overexpression of ABCA1 promoted the efflux of FC from cells to apoA-I and overexpression of ABCG1 not only promoted the FC efflux to HDL3, but also increased the CE levels in medium. A 

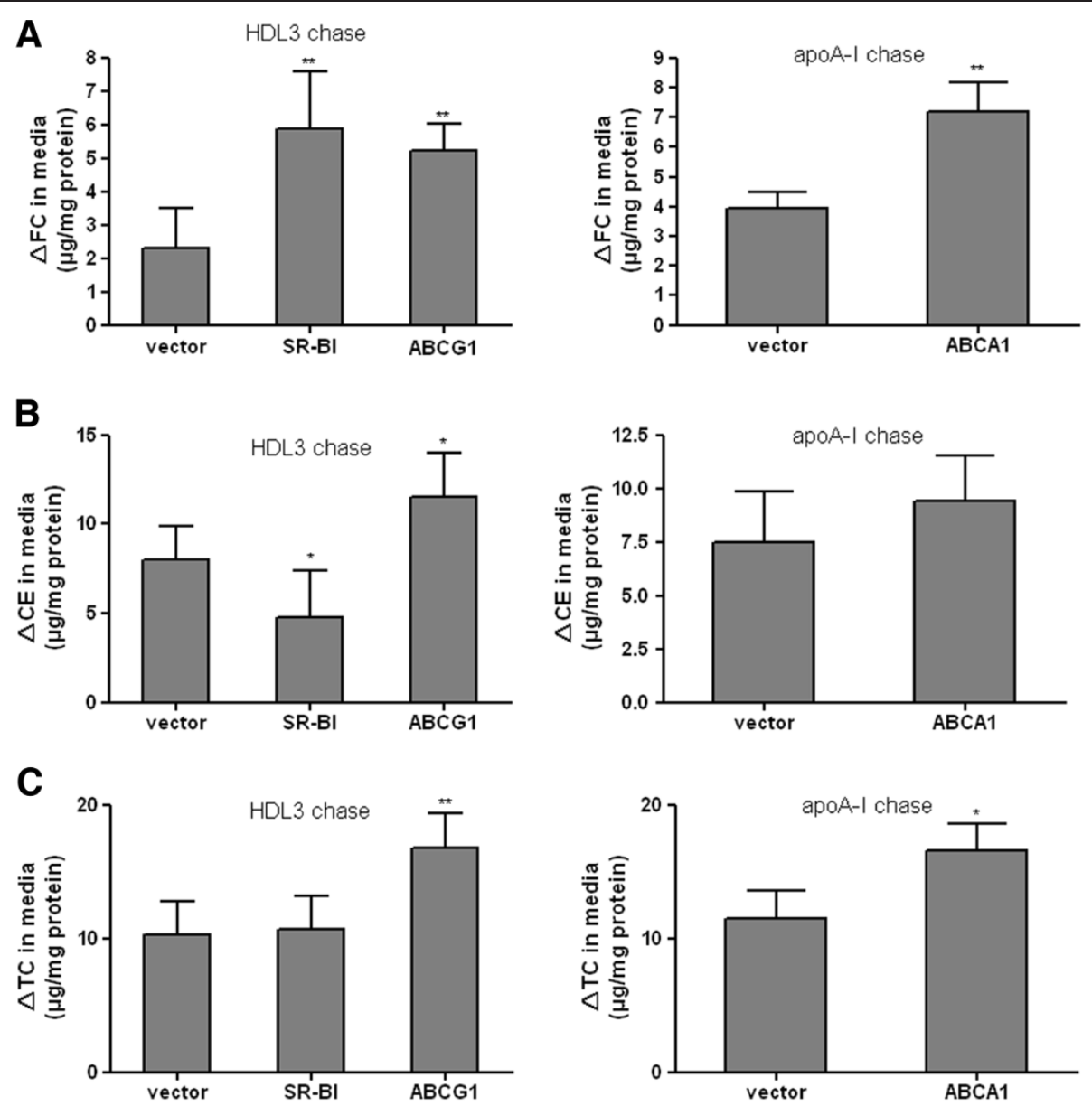

Figure 1 The single effects of SR-BI, ABCA1 and ABCG1 on cellular cholesterol efflux. CHO cells were transiently transfected with plasmid constructs expressing SR-BI, ABCA1, ABCG1 or empty vector. $24 \mathrm{~h}$ after transfection, the cells were incubated with $150 \mathrm{\mu g} / \mathrm{ml} \mathrm{HDL} 3$ (HDL3 chase) or $10 \mathrm{\mu g} / \mathrm{ml}$ apoA-I (apoA-I chase) for $24 \mathrm{~h}$ in Opti-MEM I Medium. Cholesterol efflux was determined by measuring the increased free cholesterol (FC, A), cholesteryl ester (CE, B), and total cholesterol $(T C, C)$ in medium. Values are means \pm SD of three independent experiments. ${ }^{*} p<0.05$, ${ }^{* *} p<0.01$.

previous study showed that ABCG1 increases the accumulation of $\mathrm{CE}$ in medium as a result of LCATmediated cholesterol esterification [5], so it is possible that the increase in CE induced by ABCG1 in our study might be attributable to the active LCAT, which is retained in the HDL3 particles. Previous findings have shown that ABCA1 and ABCG1 synergize to mediate cellular cholesterol export to lipoproteins [13], so we initially hypothesized that SR-BI and ABCA1 might also act synergistically on cholesterol efflux, because the nascent HDL particles formed from the ABCA1-mediated cholesterol efflux to apoA-I might serve as an acceptor for SR-BI-mediated cholesterol efflux. To assess the hypothesis, we performed cotransfection with SR-BI and ABCA1 cDNAs in $\mathrm{CHO}$ cells and then treated the cells with cholesterol acceptor apoA-I and HDL3. Out of our expectation, we found ABCA1 promoted the efflux of FC from cells, but SR-BI has no effect on ABCA1- stimulated increased FC accumulation in medium. Furthermore, we co-transfected SR-BI and ABCG1 cDNAs into $\mathrm{CHO}$ cells and found an interesting phenomenon that SR-BI inhibited ABCG1-mediated CE accumulation in medium. This was similar to a previous finding, in which it was shown SR-BI promotes cellular reuptake of the CE that initially undergoes ABCG1-mediated cholesterol efflux to HDL3 containing active LCAT [21]. In this context, we thought it is possible that SR-BI and ABCG1 might talk to each other in cells. Therefore, we performed co-immunoprecipitation experiments with cell lysates from SR-BI and ABCG1 co-transfected BHK cells to confirm whether SR-BI and ABCG1 were present in the same complex. In our expectation, we found SRBI associates with ABCG1 in transfected BHK cells. Based on our findings, it is possible that in our experimental conditions, SR-BI interacted with ABCG1 and therefore inhibited ABCG1-mediated cholesterol efflux. 


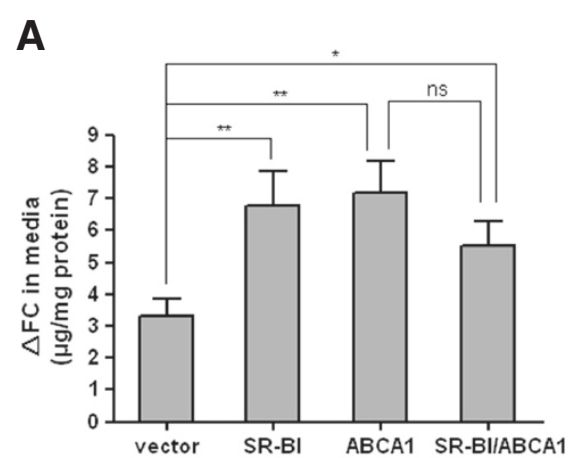

C

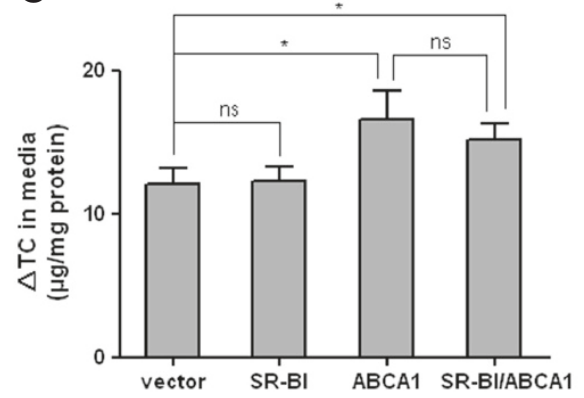

B

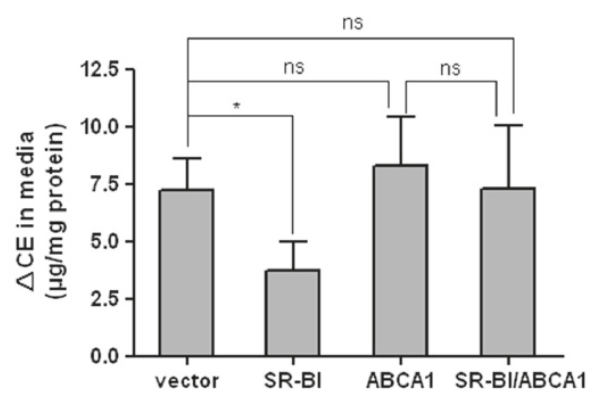

D

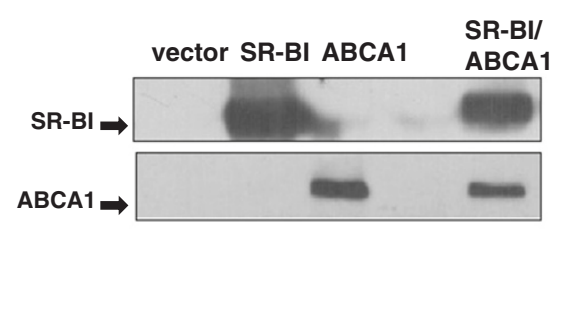

Figure 2 SR-BI does not affect ABCA1-mediated cellular cholesterol efflux. $\mathrm{CHO}$ cells were transiently transfected with SR-BI alone, ABCA1 alone, and SR-BI plus ABCA1 (co-transfection). $24 \mathrm{~h}$ after transfection, cholesterol efflux was initiated by the addition of HDL3 (150 $\mu \mathrm{g}$ protein/ml) and apoA-I $(10 \mu \mathrm{g} / \mathrm{ml})$ to medium for $24 \mathrm{~h}$. The increased FC (A), CE (B) and TC (C) mass in medium were determined. Values are means \pm SD of three independent experiments. ${ }^{*} p<0.05,{ }^{* *} p<0.01$. D shows the western blots of SR-BI and ABCA1 protein in vector transfected, SR-BI transfected, ABCA1 transfected and cotransfected cells.

The futile cycles created by the competing role of SR-BI and ABCG1 can have physiological relevance in tissues expressing $\mathrm{ABC}$ transporters, such as macrophages or liver $[16,22]$. But future studies need to be performed to confirm the effect of SR-BI on ABCA1- or ABCG1-mediated cholesterol efflux to apoA-I or HDL in macrophages and hepatocytes under basal physiological conditions because the expression levels of SR-BI and $A B C$ transporters were different among different cell types. In addition, we performed experiments in nonploarized cells, it is possible that in polarized cells, such as hepatocytes, SR-BI and ABC transporters may be expressed on different sides of the cell and the futile cycle created by the joint overexpression of SR-BI and ABCG1 in CHO cells and BHK cells might be avoided.

Besides, we also found SR-BI promoted FC efflux to HDL3, but this was counteracted by an increased uptake of CE into cells and thus no significant change in TC in medium. This was similar to previous findings that SR-BI promoted a bidirectional flux of isotopic cholesterol between cells and lipoproteins [23,24], but our study extends earlier findings suggesting that SR-BI singly does not play important roles in promoting net cholesterol efflux to HDL3. Although SR-BI did not promote net cholesterol efflux, it is possible that the cholesterol changes mediated by SR-BI may have effects on cellular cholesterol homeostasis, because CE entering the cells would not play regulatory roles corresponding to that resulting from the efflux of FC.

\section{Conclusions}

Our data suggested SR-BI associates with ABCG1 and appear to inhibit ABCG1-mediated CE accumulation in medium, but does not affect ABCA1-stimulated cholesterol efflux from cells to apoA-I or HDL3 in vitro, which might play roles in maintaining cellular cholesterol homeostasis. Further studies on the interactions between endogenous $\mathrm{SR}-\mathrm{BI}$ and $\mathrm{ABC}$ transporters in promoting cholesterol efflux in vivo need to be conducted.

\section{Methods}

Isolation of HDL from plasma

HDL3 fractions were isolated from normolipidemic human plasma by sequential ultracentrifugation at a density of $1.210 \mathrm{~g} / \mathrm{ml}$ and dialyzed against PBS containing $1 \mathrm{mM}$ EDTA [25]. The integrity of the isolated HDL3 was checked by $0.8 \%$ agarose gel electrophoresis.

\section{Cell culture and transfection}

Chinese hamster ovary $(\mathrm{CHO})$ cells purchased from ATCC were cultured in F-12 medium (Hyclone) 


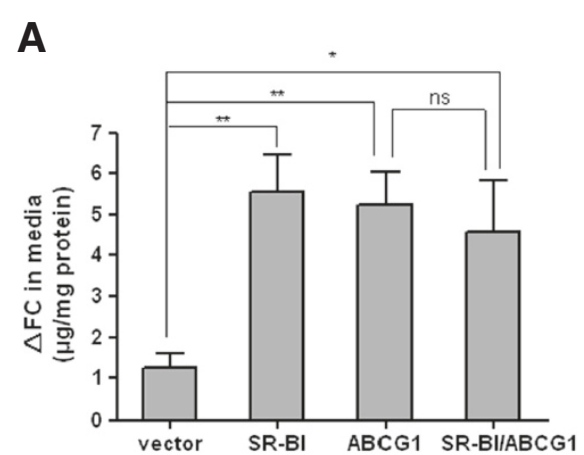

C

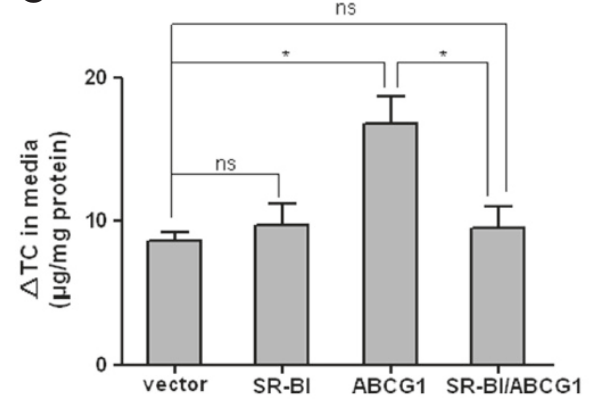

B

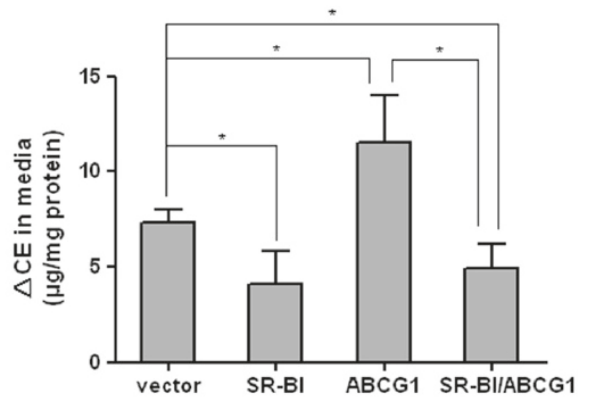

D

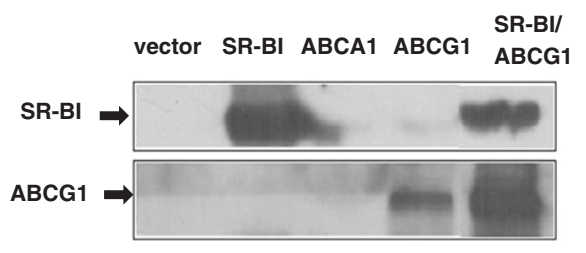

Figure 3 SR-BI inhibits ABCG1-mediated cellular cholesterol efflux. CHO cells were transiently transfected with SR-BI alone, ABCG1 alone, and SR-BI plus ABCG1 (co-transfection). $24 \mathrm{~h}$ after transfection, cholesterol efflux was initiated by the addition of HDL3 (150 $\mu \mathrm{g}$ protein/ml) to medium for $24 \mathrm{~h}$. The increased FC (A), CE (B) and TC (C) mass in medium were determined. Values are means \pm SD of three independent experiments. ${ }^{*} p<0.05,{ }^{*} p<0.01$. D shows the western blots of SR-BI and ABCG1 protein in vector transfected, SR-BI transfected, ABCG1 transfected and cotransfected cells.

supplemented with $10 \%(\mathrm{v} / \mathrm{v})$ fetal bovine serum (FBS, Gibco) and $2 \mathrm{mM}_{\mathrm{L}}$-glutamine. Cells grown to $80-90 \%$ confluence were transiently transfected with similar amounts of control empty vector (pcDNA3. $1^{+}$), murine SR-BI, ABCA1 or ABCG1 cDNA alone combined with empty vector (1:1), or both SR-BI and ABCA1 cDNA (1:1), or both SR-BI and ABCG1 cDNA (1:1) using Lipofectamine 2000 (Invitrogen) according to the manufacturer`s instructions. BHK-G (BHK cells stably expressing mifepristone inducible ABCG1) cells were kindly provided by Dr. Xian-cheng Jiang, SUNY Downstate Medical Center, USA. $10 \mathrm{nM}$ mifepristone in $1 \%$ fatty

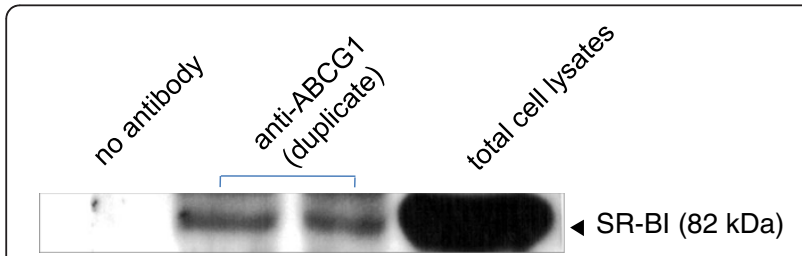

Figure 4 Co-immunoprecipitation of SR-BI with ABCG1 in BHK cells overexpressing $\mathbf{S R}-\mathbf{B I}$ and $\mathbf{A B C G} 1$. The cell lysates from the transfected BHK cells were immunoprecipitated with anti-ABCG1 antibody. The immunoprecipitates were then analyzed by western blot with antibody against SR-BI. acid-free bovine serum albumin was used for $18-20 \mathrm{hr}$ to induce ABCG1 expression. Then BHK-G cells were transiently transfected with SR-BI using Lipofectamine 2000 and incubated for $24-36 \mathrm{hr}$ prior to performing the further experiments. For all experiments, cells cultured for 4-10 passages were used.

\section{Western blot analysis}

Transfected cells were washed with phosphate-buffered saline (PBS) and lysed in lysis buffer containing $50 \mathrm{mM}$ Tris, pH 7.5, 1.0 mM EDTA, $150 \mathrm{mM} \mathrm{NaCl}, 0.1 \%$ SDS, $1 \%$ TritonX-100, $1 \%$ Sodium deoxycholate, and $1 \times$ proteinase inhibitor cocktail (Invitrogen). After heating at $95^{\circ} \mathrm{C}$ for 5 min, cell-lysate samples of equal protein concentration were subjected to SDS-PAGE and transferred onto polyvinylidene fluoride (PVDF) membranes (Millipore, USA) by electroblotting. ABCA1, ABCG1 and SR-BI proteins were detected using anti-ABCA1 polyclonal antibody (1:200 dilution, Santa Cruz Biotechnology), anti-ABCG1 polyclonal antibody (1:300, Santa Cruz Biotechnology), or anti-SR-BI monoclonal antibody (1:2000, Novus Biological Inc.). Primary antibodies were detected using a peroxidaseconjugated anti-mouse, anti-goat or anti-rabbit antibody and revealed by ECL Substrate (Pierce). 


\section{Net cholesterol efflux assays}

Cholesterol efflux assays were carried out as described with modifications [5]. In brief, 24 hours after transfection, the cells were incubated for $24 \mathrm{~h}$ in Opti-MEM serum-free medium (Invitrogen) in the presence or absence of HDL3 (150 $\mu \mathrm{g}$ protein/ml) and/or apoA-I $(10 \mu \mathrm{g} / \mathrm{ml}$, Sigma). After the incubation, the mass of cholesterol in medium was determined by using Cholesterol/Cholesteryl Ester Quantitation Kit (Biovision) according to the manufacturer's instructions. Cells were lysed and the protein content was determined by the BCA method.

\section{Co-immunoprecipitation and Western blot}

BHK cells transfected with ABCG1 and SR-BI were used for immunoprecipitation experiments. Immunoprecipitation of SR-BI and ABCG1 protein was performed by using the Protein G Immunoprecipitation Kit (Roche) according to the manufacturer's instructions. The immunoprecipitated proteins were then subjected to western blotting to analyze the target antigens in complex mixtures of proteins.

\section{Statistical analysis}

Statistical analysis was performed by one-way analysis of variance (ANOVA) test with the GraphPad Prism programme ver.4.0. Results are expressed as means \pm SD. $P$ values less than 0.05 were considered significant.

\section{Competing interests}

The authors declare that there are no conflicts of interest in our manuscript.

\section{Authors' contributions}

GS carried out the study design, data collection and analysis, and drafted the manuscript. CZ participated in the cell-based experiments and review of the manuscript. QL performed the co-immunoprecipitation experiments. YS and $J L$ conducted the isolation of HDL. WL participated in editing the manuscript. SQ and PZ was responsible for the study design, the funding, the data analysis, and the manuscript draft. All authors read and approved the final manuscript.

\section{Acknowledgements}

This research was supported by the National Natural Science Foundation of China (Grant No. 30971098; 81070247; 81170785) and by the Taishan Scholars Foundation of Shandong Province (ZD056, ZD057)

\section{Author details}

${ }^{1}$ Key Laboratory of Atherosclerosis in Universities of Shandong; Institute of Atherosclerosis, Taishan Medical University, Taian, China. ${ }^{2}$ PLA General Hospital, Beijing, China. ${ }^{3}$ 2\# YingSheng E Road, Taian, Shandong 271000, P.R. China. ${ }^{4} 28 \#$ Fuxing Road, Beijing 100853, P.R.China.

Received: 8 August 2012 Accepted: 14 September 2012

Published: 17 September 2012

\section{References}

1. Spady DK: Reverse cholesterol transport and atherosclerosis regression. Circulation 1999, 100:576-578.

2. Tall AR: An overview of reverse cholesterol transport. Eur Heart J 1998, 19(Suppl A):A31-A35.

3. Brunham LR, Kruit JK, Pape TD, Parks JS, Kuipers F, Hayden MR: Tissuespecific induction of intestinal ABCA1 expression with a liver $\mathrm{X}$ receptor agonist raises plasma HDL cholesterol levels. Circ Res 2006, 99:672-674.
4. Stangl $H$, Hyatt $M$, Hobbs HH: Transport of lipids from high and low density lipoproteins via scavenger receptor-BI. J Biol Chem 1999, 274:32692-32698.

5. Matsuura F, Wang N, Chen W, Jiang XC, Tall AR: HDL from CETP-deficient subjects shows enhanced ability to promote cholesterol efflux from macrophages in an apoE- and ABCG1-dependent pathway. J Clin Invest 2006, 116:1435-1442.

6. van Eck M, Bos IS, Kaminski WE, Orso E, Rothe G, Twisk J, Bottcher A, Van Amersfoort ES, Christiansen-Weber TA, Fung-Leung WP, et al: Leukocyte ABCA1 controls susceptibility to atherosclerosis and macrophage recruitment into tissues. Proc Natl Acad Sci U S A 2002, 99:6298-6303.

7. Van Eck M, Singaraja RR, Ye D, Hildebrand RB, James ER, Hayden MR, Van Berkel TJ: Macrophage ATP-binding cassette transporter A1 overexpression inhibits atherosclerotic lesion progression in low-density lipoprotein receptor knockout mice. Arterioscler Thromb Vasc Biol 2006, 26:929-934

8. Kennedy MA, Barrera GC, Nakamura K, Baldan A, Tarr P, Fishbein MC, Frank J, Francone OL, Edwards PA: ABCG1 has a critical role in mediating cholesterol efflux to HDL and preventing cellular lipid accumulation. Cell Metab 2005, 1:121-131

9. Baldan A, Tarr P, Vales CS, Frank J, Shimotake TK, Hawgood S, Edwards PA: Deletion of the transmembrane transporter $A B C G 1$ results in progressive pulmonary lipidosis. J Biol Chem 2006, 281:29401-29410.

10. Zhang W, Yancey PG, Su YR, Babaev VR, Zhang Y, Fazio S, Linton MF: Inactivation of macrophage scavenger receptor class B type I promotes atherosclerotic lesion development in apolipoprotein E-deficient mice. Circulation 2003, 108:2258-2263.

11. Covey SD, Krieger M, Wang W, Penman M, Trigatti BL: Scavenger receptor class B type I-mediated protection against atherosclerosis in LDL receptor-negative mice involves its expression in bone marrow-derived cells. Arterioscler Thromb Vasc Biol 2003, 23:1589-1594.

12. Van Eck M, Bos IS, Hildebrand RB, Van Rij BT, Van Berkel TJ: Dual role for scavenger receptor class $B$, type I on bone marrow-derived cells in atherosclerotic lesion development. Am J Pathol 2004, 165:785-794.

13. Gelissen IC, Harris M, Rye KA, Quinn C, Brown AJ, Kockx M, Cartland S, Packianathan $M$, Kritharides $L$, Jessup $W$ : $A B C A 1$ and $A B C G 1$ synergize to mediate cholesterol export to apoA-I. Arterioscler Thromb Vasc Biol 2006, 26:534-540.

14. Vaughan $A M$, Oram JF: $A B C A 1$ and $A B C G 1$ or $A B C G 4$ act sequentially to remove cellular cholesterol and generate cholesterol-rich HDL. J Lipid Res 2006, 47:2433-2443.

15. Yvan-Charvet L, Ranalletta M, Wang N, Han S, Terasaka N, Li R, Welch C, Tall AR: Combined deficiency of ABCA1 and ABCG1 promotes foam cell accumulation and accelerates atherosclerosis in mice. J Clin Invest 2007, 117:3900-3908.

16. Wang N, Silver DL, Costet P, Tall AR: Specific binding of ApoA-I, enhanced cholesterol efflux, and altered plasma membrane morphology in cells expressing ABC1. J Biol Chem 2000, 275:33053-33058.

17. Oram JF, Lawn RM, Garvin MR, Wade DP: ABCA1 is the CAMP-inducible apolipoprotein receptor that mediates cholesterol secretion from macrophages. J Biol Chem 2000, 275:34508-34511.

18. Bortnick AE, Rothblat GH, Stoudt G, Hoppe KL, Royer LJ, McNeish J, Francone OL: The correlation of ATP-binding cassette 1 mRNA levels with cholesterol efflux from various cell lines. J Biol Chem 2000, 275:28634-28640

19. Yancey PG, de la Llera-Moya M, Swarnakar S, Monzo P, Klein SM, Connelly MA, Johnson WJ, Williams DL, Rothblat GH: High density lipoprotein phospholipid composition is a major determinant of the bi-directional flux and net movement of cellular free cholesterol mediated by scavenger receptor BI. J Biol Chem 2000, 275:36596-36604.

20. Wang N, Lan D, Chen W, Matsuura F, Tall AR: ATP-binding cassette transporters G1 and G4 mediate cellular cholesterol efflux to highdensity lipoproteins. Proc Natl Acad Sci U S A 2004, 101:9774-9779.

21. Yvan-Charvet $L$, Pagler TA, Wang N, Senokuchi T, Brundert M, Li H, Rinninger F, Tall AR: SR-BI inhibits ABCG1-stimulated net cholesterol efflux from cells to plasma HDL. J Lipid Res 2008, 49:107-114.

22. Klucken J, Buchler C, Orso E, Kaminski WE, Porsch-Ozcurumez M, Liebisch G, Kapinsky M, Diederich W, Drobnik W, Dean M, et al: ABCG1 (ABC8), the human homolog of the Drosophila white gene, is a regulator of macrophage cholesterol and phospholipid transport. Proc Natl Acad Sci U S A 2000, 97:817-822. 
23. de la Llera-Moya M, Rothblat GH, Connelly MA, Kellner-Weibel G, Sakr SW, Phillips MC, Williams DL: Scavenger receptor BI (SR-BI) mediates free cholesterol flux independently of $\mathrm{HDL}$ tethering to the cell surface. J Lipid Res 1999, 40:575-580.

24. de La Llera-Moya M, Connelly MA, Drazul D, Klein SM, Favari E, Yancey PG, Williams DL, Rothblat GH: Scavenger receptor class B type I affects cholesterol homeostasis by magnifying cholesterol flux between cells and HDL. J Lipid Res 2001, 42:1969-1978.

25. Brundert M, Heeren J, Bahar-Bayansar M, Ewert A, Moore KJ, Rinninger F: Selective uptake of HDL cholesteryl esters and cholesterol efflux from mouse peritoneal macrophages independent of SR-BI. J Lipid Res 2006 $47: 2408-2421$

doi:10.1186/1476-511X-11-118

Cite this article as: Song et al:: SR-BI associates with ABCG1 and inhibits ABCG1-mediated cholesterol efflux from cells to high-density lipoprotein 3. Lipids in Health and Disease 2012 11:118.

\section{Submit your next manuscript to BioMed Central and take full advantage of:}

- Convenient online submission

- Thorough peer review

- No space constraints or color figure charges

- Immediate publication on acceptance

- Inclusion in PubMed, CAS, Scopus and Google Scholar

- Research which is freely available for redistribution 